\title{
Pointwise approximation by Meyer-König and Zeller operators
}

\author{
by XiaO-Ming Zeng and Jun-Ning ZhaO (Xiamen)
}

\begin{abstract}
We study the rate of pointwise convergence of Meyer-König and Zeller operators for bounded functions, and get an asymptotically optimal estimate.

1. Introduction. For a function $f$ defined on $[0,1]$, the Meyer-König and Zeller operators $M_{n}$ are given by

$$
\begin{aligned}
& M_{n}(f, x)=\sum_{k=0}^{\infty} f\left(\frac{k}{n+k}\right) m_{n k}(x), \quad 0 \leq x<1, \\
& M_{n}(f, 1)=f(1), \quad m_{n k}(x)=\left(\begin{array}{c}
n+k \\
k
\end{array}\right) x^{k}(1-x)^{n+1} .
\end{aligned}
$$
\end{abstract}

The approximation-theoretical behaviour of the operators (1), such as direct approximation, best asymptotic constants, global approximation, $L_{p^{-}}$ approximation, moment estimates, etc., has been the subject of extensive investigation (cf. $[1-3,5,9,11,12]$ ).

The rates of convergence on functions of bounded variation were obtained for various operators (see $[4,6,8,13,14]$ ). In this paper we consider the rate of convergence of the operators (1) for a more general class of functions:

$$
I_{B}=\{f \mid f \text { is bounded on }[0,1]\} \text {. }
$$

In order that our work includes the case of functions of bounded variation and gives a real improvement, we introduce the following three quantities:

$$
\Omega_{x-}\left(f, \delta_{1}\right)=\sup _{t \in\left[x-\delta_{1}, x\right]}|f(t)-f(x)|, \quad \Omega_{x+}\left(f, \delta_{2}\right)=\sup _{t \in\left[x, x+\delta_{2}\right]}|f(t)-f(x)|,
$$

2000 Mathematics Subject Classification: 41A36, 41A25, 41A10.

Key words and phrases: rate of convergence, basis functions and moments of approximation operators, asymptotically optimal. China.

Project 19871068 supported by NSFC and Fujian Provincial Science Foundation of 


$$
\Omega(x, f, \lambda)=\sup _{t \in[x-x / \lambda, x+(1-x) / \lambda]}|f(t)-f(x)|,
$$

where $f \in I_{B}, x \in[0,1]$ is fixed, $0 \leq \delta_{1} \leq x, 0 \leq \delta_{2} \leq 1-x$ and $\lambda \geq 1$. It is clear that

(i) $\Omega_{x-}\left(f, \delta_{1}\right)$ and $\Omega_{x+}\left(f, \delta_{2}\right)$ are non-decreasing in $\delta_{1}$ and in $\delta_{2}$ respectively; $\Omega(x, f, \lambda)$ is non-increasing in $\lambda$.

(ii) If $f$ is continuous at $x$, then we have $\lim _{\delta_{1} \rightarrow 0+} \Omega_{x-}\left(f, \delta_{1}\right)=0$, $\lim _{\delta_{2} \rightarrow 0+} \Omega_{x+}\left(f, \delta_{2}\right)=0$ and $\lim _{\lambda \rightarrow \infty} \Omega(x, f, \lambda)=0$.

(iii) $\Omega_{x-}\left(f, \delta_{1}\right) \leq \Omega\left(x, f, x / \delta_{1}\right)$ and $\Omega_{x+}\left(f, \delta_{2}\right) \leq \Omega\left(x, f,(1-x) / \delta_{2}\right)$.

If $f$ is of bounded variation on $[a, b]$, and $\bigvee_{a}^{b}(f)$ denotes the total variation of $f$ on $[a, b]$, then

$$
\text { (iv) } \begin{aligned}
\Omega_{x-}\left(f, \delta_{1}\right) & \leq \bigvee_{x-\delta_{1}}^{x}(f), \Omega_{x+}\left(f, \delta_{2}\right) \leq \bigvee_{x}^{x+\delta_{2}}(f), \\
\Omega(x, f, \lambda) & \leq \bigvee_{x-x / \lambda}^{x+(1-x) / \lambda}(f) .
\end{aligned}
$$

Now let us state our main result:

THEOREM. If $f$ is bounded on $[0,1]$, and $f(x+)$ and $f(x-)$ exist at a fixed point $x \in(0,1)$, then for all $n>1$ we have

$$
\begin{aligned}
\mid M_{n}(f, x)-\frac{1}{2}( & f(x+)+f(x-)) \mid \leq \frac{6}{n x+1} \sum_{k=1}^{n} \Omega\left(x, g_{x}, \sqrt{k}\right) \\
& +\frac{4}{\sqrt{n x}+1}\left(|f(x+)-f(x-)|+\varepsilon_{n}(x)|f(x)-f(x-)|\right),
\end{aligned}
$$

where

$$
\varepsilon_{n}(x)= \begin{cases}1 & \text { if } x=k^{\prime} /\left(n+k^{\prime}\right) \text { for some } k^{\prime} \in \mathbb{N}, \\ 0 & \text { if } x \neq k /(n+k) \text { for any } k \in \mathbb{N}\end{cases}
$$

and $g_{x}(t)$ is defined as

$$
g_{x}(t)= \begin{cases}f(t)-f(x+), & x<t \leq 1, \\ 0, & t=x, \\ f(t)-f(x-), & 0 \leq t<x .\end{cases}
$$

Inequality $(2)$ holds at $x=0($ resp. $x=1)$ if we set $\frac{1}{2} f(x+)+\frac{1}{2} f(x-)=f(0)$ (resp. $f(1))$.

In the last part of the paper, we shall show that our estimate is asymptotically optimal.

2. Preliminary results. We first give several preliminary results, which mainly are estimates concerning the basis functions and moments of Meyer- 
König and Zeller operators. Some results and techniques of probability theory play an important role in this section.

Lemma 1. For $n \geq 2$ and $x \in[0,1]$, we have

$$
\frac{x(1-x)^{2}}{2 n} \leq M_{n}\left((t-x)^{2}, x\right) \leq \frac{2 x(1-x)^{2}}{n},
$$

and for $x \in(0,1]$ and $n$ sufficiently large,

$$
M_{n}\left((t-x)^{4}, x\right) \leq \frac{4 x^{2}(1-x)^{4}}{n^{2}} .
$$

Proof. By [3, Lemma 2.1],

$$
\left(1+\frac{2 x}{n+2}\right) \frac{x(1-x)^{2}}{n+1} \leq M_{n}\left((t-x)^{2}, x\right) \leq\left(1+\frac{2 x}{n-1}\right) \frac{x(1-x)^{2}}{n+1},
$$

which yields (4) by a simple calculation.

In addition, for $x \in(0,1]$ and $n$ sufficiently large, by [1, p. 359, Corollary] we get by direct calculation

$$
\begin{aligned}
& M_{n}\left((t-x)^{4}, x\right) \\
& \quad=M_{n}\left(t^{4}, x\right)-4 x M_{n}\left(t^{3}, x\right)+6 x^{2} M_{n}\left(t^{2}, x\right)-4 x^{3} M_{n}(t, x)+x^{4} \\
& \quad=\frac{3 x^{2}(1-x)^{4}}{n^{2}}+\frac{x(1-x)^{2}\left(25 x^{4}-112 x^{3}+82 x^{2}-2 x+1\right)}{n^{3}}+O\left(n^{-4}\right),
\end{aligned}
$$

which yields the inequality (5).

Lemma 2. For all $k \in \mathbb{N}$ and $x \in[0,1]$, we have

$$
m_{n k}(x)<\frac{2}{1+\sqrt{n x}} .
$$

Proof. From Theorem 2 of [12] it is known that

$$
m_{n k}(x)<\frac{1}{\sqrt{2 e}} \cdot \frac{1}{\sqrt{(n+1) x}} .
$$

Since $m_{n k}(x) \leq 1$, it follows that

$$
m_{n k}(x) \sqrt{n x}+m_{n k}(x)<\frac{1}{\sqrt{2 e}}+1 .
$$

The inequality (6) is proved.

LEMMA 3. Let $\left\{\xi_{k}\right\}_{k=1}^{\infty}$ be a sequence of independent random variables with the same geometric distribution

$$
P\left(\xi_{i}=k\right)=x^{k}(1-x), \quad k \in \mathbb{N}, \quad x \in(0,1) .
$$

Then

$$
\begin{gathered}
E \xi_{1}=x /(1-x), \quad E\left(\xi_{1}-E \xi_{1}\right)^{2}=x /(1-x)^{2}, \\
E\left(\xi_{1}-E \xi_{1}\right)^{3}=\left(x^{2}+x\right) /(1-x)^{3}, \quad E\left|\xi_{1}-E \xi_{1}\right|^{3} \leq 3 x /(1-x)^{3} .
\end{gathered}
$$


Pr o of. Direct calculation gives

$$
\begin{aligned}
\sum_{k=0}^{\infty} x^{k}(1-x)=1, \quad E \xi_{1} & =\sum_{k=0}^{\infty} k x^{k}(1-x)=\frac{x}{1-x}, \\
E \xi_{1}^{2}=\sum_{k=0}^{\infty} k^{2} x^{k}(1-x) & =\frac{x^{2}+x}{(1-x)^{2}} \\
E \xi_{1}^{3}=\sum_{k=0}^{\infty} k^{3} x^{k}(1-x) & =\frac{x^{3}+4 x^{2}+x}{(1-x)^{3}} \\
E \xi_{1}^{4}=\sum_{k=0}^{\infty} k^{4} x^{k}(1-x) & =\frac{x^{4}+11 x^{3}+11 x^{2}+x}{(1-x)^{4}} .
\end{aligned}
$$

Hence it is easy to show that

$$
E\left(\xi_{1}-E \xi_{1}\right)^{2}=x /(1-x)^{2}, \quad E\left(\xi_{1}-E \xi_{1}\right)^{3}=\left(x^{2}+x\right) /(1-x)^{3},
$$

and

$$
\begin{aligned}
E\left(\xi_{1}-E \xi_{1}\right)^{4}= & E \xi_{1}^{4}-4 E \xi_{1} E \xi_{1}^{3}+6\left(E \xi_{1}\right)^{2} E \xi_{1}^{2}-4\left(E \xi_{1}\right)^{3} E \xi_{1}+\left(E \xi_{1}\right)^{4} \\
= & \frac{x^{4}+11 x^{3}+11 x^{2}+x}{(1-x)^{4}}-\frac{4 x}{1-x} \frac{x^{3}+4 x^{2}+x}{(1-x)^{3}} \\
& +\frac{6 x^{2}}{(1-x)^{2}} \frac{x(1+x)}{(1-x)^{2}}-\frac{3 x^{4}}{(1-x)^{4}} \\
= & \frac{x^{3}+7 x^{2}+x}{(1-x)^{4}} .
\end{aligned}
$$

By the Hölder inequality we get

$$
\begin{aligned}
E\left|\xi_{1}-E \xi_{1}\right|^{3} & \leq \sqrt{E\left(\xi_{1}-E \xi_{1}\right)^{4} E\left(\xi_{1}-E \xi_{1}\right)^{2}} \\
& =\sqrt{\frac{\left(x^{3}+7 x^{2}+x\right) x}{(1-x)^{4}(1-x)^{2}} \leq \frac{3 x}{(1-x)^{3}} .}
\end{aligned}
$$

The proof of Lemma 3 is complete.

Lemmas 4 and 5 below are the well-known Berry-Esseen bound and the asymptotic expression for the central limit theorem of probability theory. They can be used to get upper and lower bounds for partial sums of MeyerKönig and Zeller basis functions. Their proofs can be found in Feller [7, pp. 540-543] and Shiryayev [10, p. 432].

LEMmA 4. Let $\left\{\xi_{k}\right\}_{k=1}^{\infty}$ be a sequence of independent and identically distributed random variables with $E\left(\xi_{1}\right)=a_{1}, E\left(\xi_{1}-a_{1}\right)^{2}=\sigma^{2}>0$, $E\left|\xi_{1}-a_{1}\right|^{3}=\varrho<\infty$, and let $F_{n}$ stand for the distribution function of $\sum_{k=1}^{n}\left(\xi_{k}-a_{1}\right) /(\sigma \sqrt{n})$. Then there exists an absolute constant $C, 1 / \sqrt{2 \pi} \leq$ 
$C<0.8$, such that for all $t$ and $n$,

$$
\left|F_{n}(t)-\frac{1}{\sqrt{2 \pi}} \int_{-\infty}^{t} e^{-u^{2} / 2} d u\right|<\frac{C \varrho}{\sigma^{3} \sqrt{n}} .
$$

LEMma 5. Under the conditions of Lemma $4\left(E\left|\xi_{1}-a_{1}\right|^{3}<\infty\right.$ can be reduced to $\left.E\left(\xi_{1}-a_{1}\right)^{3}<\infty\right)$, assume $F_{n}$ to be a lattice distribution. Then at all points $t$ of the lattice we have

$$
\text { (8) } \begin{array}{r}
\frac{F_{n}(t)+F_{n}(t-)}{2}-\frac{1}{\sqrt{2 \pi}} \int_{-\infty}^{t} e^{-u^{2} / 2} d u-\frac{E\left(\xi_{1}-a_{1}\right)^{3}}{6 \sigma^{3} \sqrt{n}} \cdot \frac{1-t^{2}}{\sqrt{2 \pi}} e^{-t^{2} / 2} \\
=o\left(n^{-1 / 2}\right) .
\end{array}
$$

Lemma 6. For $x \in[0,1)$, we have

$$
\left|\sum_{k>n x /(1-x)} m_{n k}(x)-\frac{1}{2}\right| \leq \frac{4}{\sqrt{n x}+1} .
$$

Pr o of. Let $\left\{\xi_{i}\right\}_{i=1}^{\infty}$ be a sequence of independent random variables with the same geometric distribution $P\left(\xi_{i}=k\right)=x^{k}(1-x), k \in \mathbb{N}, x \in(0,1)$, and let $\eta_{n+1}=\sum_{i=1}^{n+1} \xi_{i}$. Then the probability distribution of the random variable $\eta_{n+1}$ is

$$
P\left(\eta_{n+1}=k\right)=\left(\begin{array}{c}
n+k \\
k
\end{array}\right) x^{k}(1-x)^{n+1}=m_{n k}(x) .
$$

So

$$
\begin{aligned}
\sum_{k>n x /(1-x)} m_{n k}(x) & =P\left(\eta_{n+1}>\frac{n x}{1-x}\right)=1-P\left(\eta_{n+1} \leq \frac{n x}{1-x}\right) \\
& =1-F_{n+1}\left(\frac{-\sqrt{x}}{\sqrt{n+1}}\right) .
\end{aligned}
$$

By Lemmas 3 and 4 we get

$$
\begin{aligned}
\left|\sum_{k>n x /(1-x)} m_{n k}(x)-\frac{1}{2}\right| & =\left|\frac{1}{2}-F_{n+1}\left(\frac{-\sqrt{x}}{\sqrt{n+1}}\right)\right| \\
& =\left|F_{n+1}\left(\frac{-\sqrt{x}}{\sqrt{n+1}}\right)-\frac{1}{\sqrt{2 \pi}} \int_{-\infty}^{0} e^{-u^{2} / 2} d u\right| \\
& \leq \frac{C \varrho}{\sigma^{3} \sqrt{n+1}}+\frac{1}{\sqrt{2 \pi}} \int_{-\sqrt{x} / \sqrt{n+1}}^{0} e^{-u^{2} / 2} d u \\
& <0.8 \frac{3 x}{(1-x)^{3}} \cdot \frac{(1-x)^{3}}{x^{3 / 2} \sqrt{n+1}}+\frac{1}{\sqrt{2 \pi}} \cdot \frac{\sqrt{x}}{\sqrt{n+1}} \leq \frac{3}{\sqrt{n x}},
\end{aligned}
$$

and since $\left|\sum_{k>n x /(1-x)} m_{n k}(x)-1 / 2\right| \leq 1$, we obtain (9). 
3. Proof of Theorem. For any $f \in I_{B}$, if $f(x+)$ and $f(x-)$ exist at $x$, we decompose $f$ into

$$
\begin{aligned}
f(t)= & \frac{f(x+)+f(x-)}{2}+g_{x}(t)+\frac{f(x+)-f(x-)}{2} \operatorname{sgn}(t-x) \\
& +\delta_{x}(t)\left[f(x)-\frac{f(x+)+f(x-)}{2}\right]
\end{aligned}
$$

where $g_{x}(t)$ is defined in (3) and

$$
\operatorname{sgn}(t)=\left\{\begin{array}{ll}
1, & t>0, \\
0, & t=0, \\
-1, & t<0,
\end{array} \quad \delta_{x}(t)= \begin{cases}1, & t=x, \\
0, & t \neq x .\end{cases}\right.
$$

Hence

$$
\begin{aligned}
& \text { (11) }\left|M_{n}(f, x)-\frac{1}{2} f(x+)-\frac{1}{2} f(x-)\right| \leq\left|M_{n}\left(g_{x}, x\right)\right| \\
& +\left|\frac{f(x+)-f(x-)}{2} M_{n}(\operatorname{sgn}(t-x), x)+\left[f(x)-\frac{f(x+)+f(x-)}{2}\right] M_{n}\left(\delta_{x}, x\right)\right| .
\end{aligned}
$$

Direct calculation gives

$$
M_{n}\left(\delta_{x}, x\right)=\varepsilon_{n}(x) m_{n k^{\prime}}(x)
$$

and

$$
\begin{aligned}
M_{n}(\operatorname{sgn}(t-x), x) & =\sum_{k=0}^{\infty} \operatorname{sgn}\left(\frac{k}{n+k}-x\right) m_{n k}(x) \\
& =-\sum_{k<n x /(1-x)} m_{n k}(x)+\sum_{k>n x /(1-x)} m_{n k}(x) \\
& =2 \sum_{k>n x /(1-x)} m_{n k}(x)-1+\varepsilon_{n}(x) m_{n k^{\prime}}(x),
\end{aligned}
$$

where

$$
\varepsilon_{n}(x)= \begin{cases}1 & \text { if } x=k^{\prime} /\left(n+k^{\prime}\right) \text { for some } k^{\prime} \in \mathbb{N} \\ 0 & \text { if } x \neq k /(n+k) \text { for any } k \in \mathbb{N}\end{cases}
$$

By (12), (13) and Lemmas 2, 6, we have

$$
\begin{aligned}
\mid \frac{f(x+)-f(x-)}{2} M_{n}(\operatorname{sgn}( & t-x), x) \\
+ & {\left[f(x)-\frac{f(x+)+f(x-)}{2}\right] M_{n}\left(\delta_{x}, x\right) \mid }
\end{aligned}
$$




$$
\begin{aligned}
& =\mid \frac{f(x+)-f(x-)}{2}\left[2 \sum_{k>n x /(1-x)} m_{n k}(x)-1\right] \\
& \quad+[f(x)-f(x-)] \varepsilon_{n}(x) m_{n k^{\prime}}(x) \mid \\
& \leq \frac{4}{\sqrt{n x}+1}\left(|f(x+)-f(x-)|+\varepsilon_{n}(x)|f(x)-f(x-)|\right) .
\end{aligned}
$$

Now it is clear from (11) and (14) that the Theorem will be proved if we establish that

$$
\left|M_{n}\left(g_{x}, x\right)\right| \leq \frac{6}{n x+1} \sum_{k=1}^{n} \Omega\left(x, g_{x}, \sqrt{k}\right) .
$$

Recalling the Lebesgue-Stieltjes integral representations we have

$$
M_{n}\left(g_{x}, x\right)=\int_{0}^{1} g_{x}(t) d_{t} K_{n}(x, t),
$$

where

$$
K_{n}(x, t)= \begin{cases}\sum_{k \leq n t /(1-t)} m_{n k}(x), & 0<t<1, \\ 1, & t=1, \\ 0, & t=0 .\end{cases}
$$

We decompose the integral of (16) into three parts:

$$
\int_{0}^{1} g_{x}(t) d_{t} K_{n}(x, t)=\triangle_{1, n}\left(g_{x}\right)+\triangle_{2, n}\left(g_{x}\right)+\triangle_{3, n}\left(g_{x}\right),
$$

where

$$
\begin{aligned}
& \triangle_{1, n}\left(g_{x}\right)=\int_{0}^{x-x / \sqrt{n}} g_{x}(t) d_{t} K_{n}(x, t), \\
& \triangle_{2, n}\left(g_{x}\right)=\int_{x-x / \sqrt{n}}^{x+(1-x) / \sqrt{n}} g_{x}(t) d_{t} K_{n}(x, t), \\
& \triangle_{3, n}\left(g_{x}\right)=\int_{x+(1-x) / \sqrt{n}}^{1} g_{x}(t) d_{t} K_{n}(x, t) .
\end{aligned}
$$

We shall estimate $\triangle_{1, n}\left(g_{x}\right), \triangle_{2, n}\left(g_{x}\right)$ and $\triangle_{3, n}\left(g_{x}\right)$ by the quantities $\Omega_{x-}\left(g_{x}, \delta_{1}\right), \Omega_{x+}\left(g_{x}, \delta_{2}\right)$ and $\Omega\left(x, g_{x}, \lambda\right)$ (for convenience, below we write them as $\Omega_{x-}\left(\delta_{1}\right), \Omega_{x+}\left(\delta_{2}\right)$ and $\Omega(x, \lambda)$ respectively). Firstly, for $\triangle_{2, n}\left(g_{x}\right)$ 
noting that $g_{x}(x)=0$ we have

$$
\left|\triangle_{2, n}\left(g_{x}\right)\right| \leq \int_{x-x / \sqrt{n}}^{x+(1-x) / \sqrt{n}}\left|g_{x}(t)-g_{x}(x)\right| d_{t} K_{n}(x, t) \leq \Omega(x, \sqrt{n}) .
$$

Next we estimate $\left|\triangle_{1, n}\left(g_{x}\right)\right|$. Since $\Omega_{x-}\left(\delta_{1}\right)$ is non-decreasing in $\delta_{1}$, it follows that

$$
\left|\triangle_{1, n}\left(g_{x}\right)\right|=\left|\int_{0}^{x-x / \sqrt{n}} g_{x}(t) d_{t} K_{n}(x, t)\right| \leq \int_{0}^{x-x / \sqrt{n}} \Omega_{x-}(x-t) d_{t} K_{n}(x, t) .
$$

Using partial integration with $y=x-x / \sqrt{n}$, we have

$$
\begin{aligned}
\int_{0}^{x-x / \sqrt{n}} \Omega_{x-}(x-t) d_{t} K_{n}(x, t) \leq & \Omega_{x-}(x-y) K_{n}(x, y+) \\
& +\int_{0}^{y} \widehat{K}_{n}(x, t) d\left(-\Omega_{x-}(x-t)\right),
\end{aligned}
$$

where $\widehat{K}_{n}(x, t)$ is the normalized form of $K_{n}(x, t)$. Since $\widehat{K}_{n}(x, t) \leq K_{n}(x, t)$ and $K_{n}(x, y+)=K_{n}(x, y)$ on $(0,1)$, using the inequality (4), we deduce that

$$
\begin{aligned}
\widehat{K}_{n}(x, t) & \leq K_{n}(x, t) \leq \sum_{k /(n+k) \leq t} m_{n k}(x) \\
& \leq \sum_{k /(n+k) \leq t} \frac{|k /(n+k)-x|^{2}}{(t-x)^{2}} m_{n k}(x) \leq \frac{2 x(1-x)^{2}}{n(t-x)^{2}} .
\end{aligned}
$$

From (18) it follows that

$$
\begin{aligned}
\left|\triangle_{1, n}\left(g_{x}\right)\right| \leq & \Omega_{x-}(x-y) \frac{2 x(1-x)^{2}}{n(x-y)^{2}} \\
& +\frac{2 x(1-x)^{2}}{n} \int_{0}^{y} \frac{d\left(-\Omega_{x-}(x-t)\right)}{(x-t)^{2}} .
\end{aligned}
$$

Since

$$
\begin{aligned}
& \int_{0}^{y} \frac{d\left(-\Omega_{x-}(x-t)\right)}{(x-t)^{2}} \\
& \quad=-\left.\frac{1}{(x-t)^{2}} \Omega_{x-}(x-t)\right|_{0} ^{y+}+\int_{0}^{y} \Omega_{x-}(x-t) \frac{2}{(x-t)^{3}} d t \\
& \quad=-\frac{1}{(x-t)^{2}} \Omega_{x-}(x-y)+\frac{\Omega_{x-}(x)}{x^{2}}+\int_{0}^{y} \Omega_{x-}(x-t) \frac{2}{(x-t)^{3}} d t
\end{aligned}
$$


from (19) we have

$\left|\triangle_{1, n}\left(g_{x}\right)\right| \leq \frac{2 x(1-x)^{2}}{n x^{2}} \Omega_{x-}(x)+\frac{2 x(1-x)^{2}}{n} \int_{0}^{x-x / \sqrt{n}} \Omega_{x-}(x-t) \frac{2}{(x-t)^{3}} d t$.

Putting $t=x-x / \sqrt{u}$ in the last integral we get

$$
\int_{0}^{x-x / \sqrt{n}} \Omega_{x-}(x-t) \frac{2}{(x-t)^{3}} d t=\frac{1}{x^{2}} \int_{1}^{n} \Omega_{x-}(x / \sqrt{u}) d u .
$$

Consequently,

$$
\left|\triangle_{1, n}\left(g_{x}\right)\right| \leq \frac{2(1-x)^{2}}{n x}\left(\Omega_{x-}(x)+\int_{1}^{n} \Omega_{x-}(x / \sqrt{u}) d u\right) .
$$

Using a similar method for estimating $\left|\triangle_{3, n}\left(g_{x}\right)\right|$ we get

$$
\left|\triangle_{3, n}\left(g_{x}\right)\right| \leq \frac{2 x^{2}}{n x}\left(\Omega_{x+}(1-x)+\int_{1}^{n} \Omega_{x+}((1-x) / \sqrt{u}) d u\right) .
$$

From (17), (20) and (21) it follows that

(22) $\left|M_{n}\left(g_{x}, x\right)\right| \leq \Omega(x, \sqrt{n})$

$$
+\left(\frac{2(1-x)^{2}}{n x}+\frac{2 x^{2}}{n x}\right)\left(\Omega(x, 1)+\int_{1}^{n} \Omega(x, \sqrt{u}) d u\right) .
$$

By the monotonicity of $\Omega(x, \lambda)$ and noting that $(1-x)^{2}+x^{2} \leq 1$, from $(22)$ we get

$$
\text { (23) } \begin{aligned}
\left|M_{n}\left(g_{x}, x\right)\right| & \leq \frac{1}{n} \sum_{k=1}^{n} \Omega(x, \sqrt{k})+\frac{2}{n x}\left(\sum_{k=1}^{n} \Omega(x, \sqrt{k})+\sum_{k=1}^{n} \Omega(x, \sqrt{k})\right) \\
& \leq \frac{5}{n x} \sum_{k=1}^{n} \Omega(x, \sqrt{k}) .
\end{aligned}
$$

On the other hand

$$
\begin{aligned}
\left|M_{n}\left(g_{x}, x\right)\right| & =\left|\int_{0}^{1}\left(g_{x}(t)-g_{x}(x)\right) d_{t} K_{n}(x, t)\right| \\
& \leq \Omega(x, 1) \leq \sum_{k=1}^{n} \Omega(x, \sqrt{k}) .
\end{aligned}
$$

The inequality (15) now follows from (23) and (24). The proof of the Theorem is complete.

4. Asymptotic optimality of our estimate. We now show that our estimate (2) is asympototically optimal. For $f \in I_{B}$, if $x$ is a continuity 
point of $f$, then (2) becomes

$$
\left|M_{n}(f, x)-f(x)\right| \leq \frac{6}{n x+1} \sum_{k=1}^{n} \Omega(x, f, \sqrt{k}) .
$$

Taking the function $f_{x}(t)=|t-x|$, from (25) we have

$$
\begin{aligned}
\left|M_{n}\left(f_{x}, x\right)-f_{x}(x)\right| & =M_{n}(|t-x|, x) \\
& \leq \frac{6}{n x+1} \sum_{k=1}^{n} \frac{1}{\sqrt{k}} \leq \frac{12}{x \sqrt{n}+1 / \sqrt{n}} .
\end{aligned}
$$

On the other hand, for any small positive number $\delta$, it is easy to show that

$$
\begin{aligned}
& \delta \sum_{k=0}^{\infty}\left|\frac{k}{n+k}-x\right| m_{n k}(x)+\frac{1}{\delta^{2}} \sum_{k=0}^{\infty}\left(\frac{k}{n+k}-x\right)^{4} m_{n k}(x) \\
& \geq \sum_{|k /(n+k)-x| \leq \delta}\left(\frac{k}{n+k}-x\right)^{2} m_{n k}(x)+\sum_{|k /(n+k)-x|>\delta}\left(\frac{k}{n+k}-x\right)^{2} m_{n k}(x) \\
& =\sum_{k=0}^{\infty}\left(\frac{k}{n+k}-x\right)^{2} m_{n k}(x) .
\end{aligned}
$$

That is,

$$
\begin{aligned}
\sum_{k=0}^{\infty}\left|\frac{k}{n+k}-x\right| m_{n k}(x) \geq & \frac{1}{\delta} \sum_{k=0}^{\infty}\left(\frac{k}{n+k}-x\right)^{2} m_{n k}(x) \\
& -\frac{1}{\delta^{3}} \sum_{k=0}^{\infty}\left(\frac{k}{n+k}-x\right)^{4} m_{n k}(x) .
\end{aligned}
$$

Hence, from Lemma 1 for $n$ sufficiently large, it follows that

$$
\sum_{k=0}^{\infty}\left|\frac{k}{n+k}-x\right| m_{n k}(x) \geq \frac{x(1-x)^{2}}{2 n \delta}-\frac{4}{\delta^{3}} \frac{x^{2}(1-x)^{4}}{n^{2}} .
$$

Choose $\delta=4 \sqrt{x(1-x)^{2} / n}$ to get

$$
M_{n}(|t-x|, x)=\sum_{k=0}^{\infty}\left|\frac{k}{n+k}-x\right| m_{n k}(x) \geq \frac{1}{16} \frac{\sqrt{x(1-x)^{2}}}{\sqrt{n}} .
$$

Therefore from (26) and (27) we see that (25) cannot be asymptotically improved.

To prove that the second term on the right hand side of (2) is asymptotically optimal, one needs an accurate estimate. If $g_{x} \equiv 0$, then (2) becomes 
(28)

$$
\begin{array}{r}
\left|M_{n}(f, x)-\frac{f(x+)+f(x-)}{2}\right| \\
\leq \frac{4\left(|f(x+)-f(x-)|+\varepsilon_{n}(x)|f(x)-f(x-)|\right)}{\sqrt{n x}+1} .
\end{array}
$$

We consider the function

$$
f(t)= \begin{cases}1, & 0 \leq t<1 / 2 \\ 0, & 1 / 2 \leq t \leq 1\end{cases}
$$

at the point $t=1 / 2$. Then

$$
\left|M_{n}(f, 1 / 2)-\frac{1}{2}\left(f\left(\frac{1}{2}+\right)+f\left(\frac{1}{2}-\right)\right)\right|=\left|\sum_{k<n} m_{n k}(1 / 2)-\frac{1}{2}\right| .
$$

From Lemma 5 and a simple calculation for geometric distributions it follows that

$$
\frac{1}{2}\left[F_{n+1}(0)+F_{n+1}(0-)\right]-\frac{1}{2}-\frac{1+x}{6 \sqrt{n+1} \sqrt{x}} \cdot \frac{1}{\sqrt{2 \pi}}=o(1 / \sqrt{n+1}) .
$$

That is,

$$
\begin{array}{r}
\frac{1}{2}\left[\sum_{k \leq \frac{(n+1) x}{1-x}} m_{n k}(x)+\sum_{k<\frac{(n+1) x}{1-x}} m_{n k}(x)\right]-\frac{1}{2}-\frac{1+x}{6 \sqrt{n+1} \sqrt{x}} \cdot \frac{1}{\sqrt{2 \pi}} \\
=o(1 / \sqrt{n+1}) .
\end{array}
$$

Taking $x=1 / 2$ in $(29)$ we get

$$
\begin{aligned}
\sum_{k<n} m_{n k} & (1 / 2)-1 / 2 \\
= & \frac{1}{4 \sqrt{\pi} \sqrt{n+1}}-m_{n n}(1 / 2)-\frac{1}{2} m_{n, n+1}(1 / 2)+o(1 / \sqrt{n+1}) .
\end{aligned}
$$

Using Stirling's formula $n !=(2 \pi n)^{1 / 2}(n / e)^{n} e^{\theta_{n} /(12 n)}\left(0<\theta_{n}<1\right)$, we find that

$$
\frac{1}{4 \sqrt{\pi} \sqrt{n+1}}-\frac{1}{2} m_{n, n+1}(1 / 2)=o(1 / \sqrt{n+1})
$$

and

$$
\frac{1}{5 \sqrt{n}}<m_{n n}(1 / 2)=\frac{(2 n) !}{(n !)^{2}}(1 / 2)^{2 n+1}=\frac{e^{\theta_{2 n} /(24 n)}}{2 \sqrt{\pi} e^{\theta_{n} /(6 n)}} \cdot \frac{1}{\sqrt{n}}<\frac{1}{2 \sqrt{n}} .
$$

Consequently, for $n$ sufficiently large,

$$
\begin{aligned}
\frac{1}{6 \sqrt{n}} & \leq\left|M_{n}(f, 1 / 2)-\frac{1}{2}\left(f\left(\frac{1}{2}+\right)+f\left(\frac{1}{2}-\right)\right)\right| \\
& =\left|\sum_{k<n} m_{n k}(1 / 2)-1 / 2\right| \leq \frac{1}{\sqrt{n}}
\end{aligned}
$$

Therefore (28) cannot be asymptotically improved as $n \rightarrow+\infty$. 


\section{References}

[1] U. Abel, The moments for the Meyer-König and Zeller operators, J. Approx. Theory 82 (1995), 352-361.

[2] J. A. H. Alkemade, The second moment for the Meyer-König and Zeller operators, ibid. 40 (1984), 261-273.

[3] M. Becker and R. J. Nessel, A global approximation theorem for the Meyer-König and Zeller operators, Math. Z. 160 (1978), 195-206.

[4] R. Bojanic and M. Vuilleumier, On the rate of convergence of Fourier-Legendre series of functions of bounded variation, J. Approx. Theory 31 (1981), 67-79.

[5] E. W. Cheney and A. Sharma, Bernstein power series, Canad. J. Math. 16 (1964), 241-252.

[6] F. Cheng, On the rate of convergence of Bernstein polynomials of functions of bounded variation, J. Approx. Theory 39 (1983), 259-274.

[7] W. Feller, An Introduction to Probability Theory and Its Applications, Wiley, New York, 1971.

[8] S. Guo and M. Khan, On the rate of convergence of some operators on functions of bounded variation, J. Approx. Theory 58 (1989), 90-101.

[9] V. Maier, M. W. Müller and J. Swetits, L $L_{1}$ saturation class of the integrated Meyer-König and Zeller operators, ibid. 32 (1981), 27-31.

[10] A. N. Shiryayev, Probability, Springer, New York, 1984.

[11] V. Totik, Approximation by Meyer-König and Zeller type operators, Math. Z. 182 (1983), 425-446.

[12] X. M. Zeng, Bounds for Bernstein basis functions and Meyer-König and Zeller basis functions, J. Math. Anal. Appl. 219 (1998), 364-376.

[13] - On the rate of convergence of the generalized Szász type operators for bounded variation functions, ibid. 226 (1998), 309-325.

[14] X. M. Zeng and A. Piriou, On the rate of convergence of two Bernstein-Bézier type operators for bounded variation functions, J. Approx. Theory 95 (1998), 369-387.

Department of Mathematics

Xiamen University

Xiamen 361005

People's Republic of China

E-mail: xmzeng@jingxian.xmu.edu.cn 